\title{
OPTIMAL PORTFOLIO SELECTION WITH TRANSACTION COSTS *
}

\author{
Phillip Collings and Ulrich G. Haussmann \\ Department of Mathematics, University of British Columbia \\ 1984 Mathematics Road, Vancouver, B.C., Canada, V6T $1 Z 2$.
}

uhaus@math.ubc.ca

\section{INTRODUCTION}

Consider an investor who has the following instruments available to him: a bank account paying a fixed rate of interest $r$ and $n$ risky assets ("stocks") whose prices are modeled as geometric Brownian motions. The investor is allowed to consume at a rate $c(t)$ from the bank account and is subject to the constraint that he remain solvent at all times. Any trading in the stocks must be selffinancing, and incurs a transaction cost which is proportional to the amount being traded. The investor's objective is to maximize his expected discounted utility of lifetime consumption.

Similar problems were first studied by Constantinides [2] and Magill and Constantinides [9] for $n=1$, and solved by Davis and Norman [3]. Technically speaking, the optimal process is a reflecting diffusion inside the no trade region (a wedge) and the buying and selling strategies $L_{1}(t)$ and $M_{1}(t)$, cf below, are local times at the boundaries.

Akian, Menaldi and Sulem [1] consider the multi-asset case described above $(n>1)$, assuming that the noise terms are uncorrelated. Using dynamic programming methods, the value function is shown to be the unique viscosity solution of a variational inequality. This inequality is then discretized and solved numerically to provide the optimal strategy and indicate the shape of the trading boundaries, but convergence is not proved.

We present a different numerical scheme based on some ideas of Kushner and Martin [8], Kushner and Dupuis [7] and Fleming and Fitzpatrick [4], and we show convergence of the value functions.

Section 2 deals with the formulation and theoretical study of the portfolio selection problem. We introduce the model employed by Akian et al. [1], but

\footnotetext{
*This work was supported by the Natural Sciences and Engineering Research Council of Canada under Grant 88051.
}

\footnotetext{
The original version of this chapter was revised: The copyright line was incorrect. This has been corrected. The Erratum to this chapter is available at DOI: 10.1007/978-0-387-35359-3_40 
with correlated noise, and we formulate the control problem associated with the model. The analytical methods of [1] carry over without much difficulty to show that the value function is the unique viscosity solution of a variational inequality. Then we transform the singular control problem into a new problem involving only absolutely continuous controls, using a random time change. It is shown that the value functions for the two problems are closely related and that the value function for the transformed problem is the unique viscosity solution of the corresponding Hamilton-Jacobi-Bellman (H.J.B) equation.

The Markov chain approximation to the transformed problem is constructed in Section 3, and convergence of the the value functions of the transformed problem is established as the discretization parameter goes to zero. Although the discretization method follows that of Kushner et al, [8], [7], the convergence arguments do not. They rely on the viscosity solution techniques used by Fitzpatrick and Fleming, [4].

Details will be given in the forthcoming thesis of the first author.

\section{PROBLEM FORMULATION AND ANALYSIS}

Let $(\Omega, \mathcal{F}, P)$ be a probability space with a given filtration $\left(\mathcal{F}_{t}\right)_{t \geq 0}$. We let the processes $S_{0}(t)$ and $S_{i}(t)$ be the amount of money invested in the bank account and the $i^{\text {th }}$ risky asset at time $t$ respectively. Then

$$
\begin{aligned}
d S_{0}(t) & =\left[r S_{0}(t)-c(t)\right] d t+\sum_{i=1}^{n}\left[-\left(1+\lambda_{i}\right) d L_{i}(t)+\left(1-\mu_{i}\right) d M_{i}(t)\right] \\
d S_{i}(t) & =b_{i} S_{i}(t) d t+\sum_{j=1}^{n} \sigma_{i j} S_{i}(t) d W_{j}(t)+d L_{i}(t)-d M_{i}(t) \\
S_{i}(0) & =x_{i}, \quad i=0, \cdots, n
\end{aligned}
$$

where $W_{j}(t), j=1, \cdots, n$, are independent Brownian motions and $L_{i}(t)$ and $M_{i}(t)$ represent the cumulative purchase and sale of the $i^{t h}$ risky asset on $[0, t]$ respectively. The constants $r$ and $b_{i}$ represent the interest rate and the mean return for stock $i$ respectively and $\sigma \equiv\left(\sigma_{i, j}\right)_{i, j=1, \ldots, n}$ is the volatility matrix which measures the level of "noisiness" of the stock price processes. The coefficients $\lambda_{i}$ and $\mu_{i}$ are the proportionality constants of the transaction costs associated with stock $i$, so that purchasing 1 dollar's worth of asset $i$ will cost $\left(1+\lambda_{i}\right)$ dollars, which is transferred from the bank account. Conversely selling 1 dollar's worth of stock $i$ will result in a payment of $\left(1-\mu_{i}\right)$ dollars into the bank account.

Definition 2.1 A policy for investment and consumption is a vector $\left(c(t),\left(L_{i}(t), M_{i}(t)\right)_{i=1, \cdots, n}\right)$ of measurable adapted processes such that

1. $c(t) \geq 0$ and $\int_{0}^{t} c(\theta) d \theta<\infty$ for a.e $(t, \omega)$,

2. $L_{i}(t)$ and $M_{i}(t)$ are non decreasing, left continuous processes with right limits and $L_{i}(0)=M_{i}(0)=0$. 
For a policy $\mathcal{P}:=\left(c,\left(L_{i}, M_{i}\right)_{i=1, \cdots, n}\right)$, the corresponding processes $S_{0}(t)$ and $S_{i}(t)$ are left continuous with right hand limits and (2.1) is equivalent to

$$
\begin{aligned}
& S_{0}(t)=x_{0}+\int_{0}^{t}\left[r S_{0}(\theta)-c(\theta)\right] d \theta+\sum_{i=1}^{n}\left[-\left(1+\lambda_{i}\right) L_{i}(t)+\left(1-\mu_{i}\right) M_{i}(t)\right], \\
& S_{i}(t)=x_{i}+\int_{0}^{t} b_{i} S_{i}(\theta) d \theta+\sum_{j=1}^{n} \int_{0}^{t} \sigma_{i j} S_{i}(\theta) d W_{j}(\theta)+L_{i}(t)-M_{i}(t), \quad(2.2)
\end{aligned}
$$

for $t \geq 0$. We define the solvency region to be

$$
\mathcal{S}=\left\{x=\left(x_{0}, x_{1}, \cdots, x_{n}\right): \mathcal{W}(x)>0\right\}
$$

where

$$
\left.\mathcal{W}(x)=x_{0}+\sum_{i=1}^{n} \min \left\{\left(1-\mu_{i}\right) x_{i},\left(1+\lambda_{i}\right) x_{i}\right)\right\}
$$

Note that $\mathcal{W}(x)$ represents the the net wealth, that is the amount of money in the bank account after the investor has liquidated all her assets.

Definition 2.2 A policy $\mathcal{P} \equiv\left(c(t),\left(L_{i}(t), M_{i}(t)\right)_{i=1, \cdots, n}\right)$ is admissible if $S(t) \in$ $\overline{\mathcal{S}}$ a.s. $t \geq 0$, i.e.

$$
\mathcal{W}(S(t)) \geq 0 \quad \text { a.s. } t \geq 0 .
$$

Denote by $\mathcal{U}(x)$ the set of all admissible polices. Observe that when $S(t) \in$ $\partial \mathcal{S}$, the only admissible action is to trade to zero, i.e. $S(t+)=0$ with no consumption. Thereafter $S=0$. For $x \in \overline{\mathcal{S}}$ and $\mathcal{P} \in \mathcal{U}(x)$, define

$$
J(x, \mathcal{P})=E_{x} \int_{0}^{\infty} e^{-\delta t} \frac{c(t)^{\gamma}}{\gamma} d t
$$

where $E_{x}$ is the expectation operator given an initial endowment $x, \delta$ is a positive discount factor and $0<\gamma<1$. Here (2.5) represents the investor's expected discounted utility of lifetime consumption. The problem is to find

$$
V(x):=\sup _{\mathcal{P} \in \mathcal{U}(x)} J(x, \mathcal{P}) .
$$

We now make the following assumptions:

- [A.1] $\sigma$ is invertible and

$$
\delta>\gamma\left(r+\frac{1}{2(1-\gamma)}(\mathbf{b}-r \mathbf{1})^{T} \mathbf{a}^{-1}(\mathbf{b}-r \mathbf{1})\right),
$$

where $\mathbf{b}=\left(b_{1}, \cdots, b_{n}\right)^{T}, \mathbf{1}=(1, \cdots, 1)^{T}$ and $\mathbf{a}=\sigma \sigma^{T}$. 
- $\quad$ A.2] $\mu_{i}, \lambda_{i} \in[0,1), \lambda_{i}+\mu_{i}>0 \quad \forall i=1, \cdots, n$.

Remark 2.1 Assumption [A.1] reduces to the one stated in Akian, Menaldi and Sulem [1] when a is diagonal. When transaction costs are equal to zero (hence here also), assumption [A.1] implies that the value function for the problem is finite, cf. Karatzas, Lechoczky, Sethi and Shreve [6].

Let

$$
\mathcal{G}=\left\{f \in C\left(\mathbb{R}^{n}\right): \sup _{x} \frac{\mid f(x \mid}{1+\|x\|^{\gamma}}<\infty\right\}
$$

The following result can be proved as in [1].

Theorem 2.1 Suppose that assumptions [A.1] and [A.2] hold. Then

(i) the value function $V$ is concave and $\gamma$-Hölder continuous on $\overline{\mathcal{S}}$, and

(ii) $V$ is the unique viscosity solution in $\mathcal{G}$ of the variational inequality

$$
\begin{array}{r}
\max \left\{A V+G\left(\frac{\partial V}{\partial x_{0}}\right), \max _{1 \leq i \leq n} \mathcal{L}_{i} V, \max _{1 \leq i \leq n} \mathcal{M}_{i} V\right\}=0 \quad \text { in } \mathcal{S} \\
V=0 \quad \text { in } \partial \mathcal{S}
\end{array}
$$

where

$$
\begin{aligned}
A V=\frac{1}{2} \sum_{i=1}^{n} \sum_{i=1}^{n} a_{i j} x_{i} x_{j} \frac{\partial^{2} V}{\partial x_{i} x_{j}}+\sum_{i=1}^{n} b_{i} x_{i} \frac{\partial V}{\partial x_{i}}+r x_{0} \frac{\partial V}{\partial x_{0}}-\delta V, \\
\mathcal{L}_{i} V=-\left(1+\lambda_{i}\right) \frac{\partial V}{\partial x_{0}}+\frac{\partial V}{\partial x_{i}}, \\
\mathcal{M}_{i} V=\left(1-\mu_{i}\right) \frac{\partial V}{\partial x_{0}}-\frac{\partial V}{\partial x_{i}}, \\
G(p)=\max _{c \geq 0}\left(-c p+\frac{c^{\gamma}}{\gamma}\right) \\
=\left(\frac{1}{\gamma}-1\right) p^{\gamma /(\gamma-1)}
\end{aligned}
$$

and $a_{i j}$ is the $(i, j)^{\text {th }}$ entry of the matrix $\mathbf{a}$.

Using a time change (stretching time when trades occur) and adding another state and control variable, we can transform the above singular problem into a problem involving only absolutely continuous controls, cf. Kushner and Martin 
[8], Kushner and Dupuis [7] and Haussmann and Suo [5]. Let

$$
\begin{aligned}
\hat{S}_{0}(t)=x_{0} & +\int_{0}^{t}\left\{\left[r \hat{S}_{0}(\theta)-\hat{c}(\theta)\right] \hat{u}(\theta)\right. \\
& \left.+\sum_{i=1}^{n}\left[-\left(1+\lambda_{i}\right) \hat{l}_{i}(\theta)+\left(1-\mu_{i}\right) \hat{m}_{i}(\theta)\right](1-\hat{u}(\theta))\right\} d \theta \\
\hat{S}_{i}(t)=x_{i} & +\int_{0}^{t}\left\{b_{i} \hat{S}_{i}(\theta) \hat{u}(\theta)+\left(\hat{l}_{i}(\theta)-\hat{m}_{i}(\theta)\right)(1-\hat{u}(\theta))\right\} d \theta \\
& +\sum_{j=1}^{n} \int_{0}^{t} \sigma_{i j} \hat{S}_{i}(\theta) \sqrt{\hat{u}(\theta)} d \hat{W}_{j}(\theta), \quad i=1, \cdots, n, \\
\hat{S}_{n+1}(t)=x_{n+1} & +\int_{0}^{t} \hat{u}(\theta) d \theta .
\end{aligned}
$$

The control variable for this problem is given by a vector of measurable, adapted processes, $\hat{\mathcal{P}} \equiv\left(\hat{c}(t),\left(\hat{l}_{i}(t), \hat{m}_{i}(t)\right)_{i=1, \cdots, n}, \hat{u}(t)\right)$, taking values in $\mathbb{R}_{+} \times$ $\mathcal{Z} \times[0,1]$, where $\mathcal{Z}=\left\{z \in \mathbb{R}_{+}^{2 n}: \sum_{i=1}^{2 n} z_{i}=1\right\}$. Note that $\hat{l}_{i}\left(\hat{m}_{i}\right)$ is the rate at which stock $i$ is bought (sold), and $\hat{u}=0$ when trading occurs. Define

$$
\hat{\mathcal{S}}=\mathcal{S} \times \mathbb{R},
$$

where $\mathcal{S}$ is defined by $(2.3)$.

Definition 2.3 A control $\hat{\mathcal{P}} \equiv\left(\hat{c}(\cdot),\left(\hat{l}_{i}(\cdot), \hat{m}_{i}(\cdot)\right)_{i=1, \cdots, n}, \hat{u}(\cdot)\right)$ is said to be admissible for the transformed problem if

$$
\hat{S}(t) \in \overline{\hat{\mathcal{S}}} \quad \text { a.s. } t \geq 0 .
$$

Let $\hat{\mathcal{U}}\left(x, x_{n+1}\right)$ be the set of admissible polices for the transformed problem. Observe that it is independent of $x_{n+1}$, and as before, if $\hat{S}(t) \in \partial \hat{S}$, then the only admissible action is to take $\hat{u}=0$ and to trade (continuously) to zero. Given the initial endowment $\hat{x}:=\left(x, x_{n+1}\right) \in \hat{\mathcal{S}}$ and $\hat{\mathcal{P}} \in \hat{\mathcal{U}}(\hat{x})$, define

$$
\hat{J}(\hat{x}, \hat{\mathcal{P}})=\hat{E}_{\hat{x}} \int_{0}^{\infty} e^{-\delta \hat{S}_{n+1}(t)} \frac{\hat{c}(t)^{\gamma}}{\gamma} \hat{u}(t) d t
$$

where $\hat{E}_{\hat{x}}$ is the expectation operator with respect to the new probability measure $\hat{P}$ given the initial endowment $\hat{x}$. The transformed problem is to find

$$
\hat{V}(\hat{x}):=\sup _{\hat{P} \in \hat{\mathcal{U}}(\hat{x})} \hat{J}(\hat{x}, \hat{\mathcal{P}}) .
$$

It can be shown as in [5] that the two control problems are equivalent, that is Proposition 2.1

$$
\hat{V}(\hat{x})=e^{-\delta x_{n+1}} V(x) \equiv e^{-\delta x_{n+1}} V\left(x_{0}, \cdots, x_{n}\right) .
$$


The Hamilton-Jacobi-Bellman equation corresponding to problem (2.14) is

$$
\begin{aligned}
\max _{(\hat{l}, \hat{m}, \hat{u}) \in \mathcal{Z} \times[0,1]} & \left\{\hat{u} \hat{A} \hat{V}(x)+\hat{u} \exp \left(\frac{\delta x_{n+1}}{\gamma-1}\right) G\left(\frac{\partial \hat{V}(x)}{\partial x_{0}}\right)\right. \\
\left.+(1-\hat{u}) \sum_{i=1}^{n}\left[\left(\mathcal{L}_{i} \hat{V}(x)\right) \hat{l}_{i}+\left(\mathcal{M}_{i} \hat{V}(x)\right) \hat{m}_{i}\right]\right\}=0 \quad x \in \hat{\mathcal{S}} & \hat{V}(x)=0 \quad x \in \partial \hat{\mathcal{S}}
\end{aligned}
$$

where

$$
\hat{A} \hat{V}=\frac{1}{2} \sum_{i=1}^{n} \sum_{j=1}^{n} a_{i j} x_{i} x_{j} \frac{\partial^{2} \hat{V}}{\partial x_{i} x_{j}}+\sum_{i=1}^{n} b_{i} x_{i} \frac{\partial \hat{V}}{\partial x_{i}}+r x_{0} \frac{\partial \hat{V}}{\partial x_{0}}+\frac{\partial \hat{V}}{\partial x_{n+1}}
$$

Let $\hat{\mathcal{G}}:=\left\{e^{-\delta x_{n+1}} f: f \in \mathcal{G}\right\} \subset C\left(\mathbb{R}^{n+1}\right)$. Then we have

Theorem 2.2 Assume [A.1], [A.2]. The value function $\hat{V}$ is the unique viscosity solution in $\hat{\mathcal{G}}$ of (2.15).

\section{MARKOV CHAIN APPROXIMATION}

Let $h>0$ be an approximation parameter and define $\Sigma^{h}$ to be the lattice

$$
\begin{array}{r}
\Sigma^{h}=\left\{\hat{x} \in \mathbb{R}^{n+2}: \hat{x}=h \sum_{j=0}^{n+1} e_{j} K_{j}, \quad K_{j}=0, \pm 1, \pm 2, \cdots, j=0, \cdots, n,\right. \\
\left.K_{n+1}=0,1, \cdots\right\}
\end{array}
$$

and $\Sigma_{N}^{h}=\left\{\hat{x} \in \Sigma^{h}: x_{i} \leq N h\right\}$, where $N$ is some positive integer which will be chosen so that $N h \rightarrow \infty$ as $h \rightarrow 0$. Define the set $\hat{\mathcal{S}}_{N}^{h}$, the boundary $\partial \hat{\mathcal{S}}_{N}^{h}$ and the outer boundary $\partial \hat{\mathcal{S}}_{N}^{h+}$ to be

$$
\begin{aligned}
\hat{\mathcal{S}}_{N}^{h} & =\hat{\mathcal{S}} \cap \Sigma_{N}^{h} \\
\partial \hat{\mathcal{S}}_{N}^{h} & =\Sigma_{N}^{h} \cap\left(\left[\partial \hat{\mathcal{S}} \pm e_{i} h \pm e_{j} h\right] \backslash \hat{\mathcal{S}} \quad i=0, \cdots, n\right) . \\
\partial \hat{\mathcal{S}}_{N}^{h+} & =\left\{x \in \hat{\mathcal{S}}_{N+1}^{h}: x_{i}=(N+1) h \quad \text { for at least one } i=0, \cdots, n+1\right\} .
\end{aligned}
$$

We will now construct a Markov chain defined on $\hat{\mathcal{H}}_{N}^{h}:=\hat{\mathcal{S}}_{N}^{h} \cup \partial \hat{\mathcal{S}}_{N}^{h} \cup \partial \hat{\mathcal{S}}_{N}^{h+}$ whose behaviour "closely matches" that of the continuous process $\hat{S}$.

Define $\hat{U}_{N}^{h}:=[0, K N h] \times \mathcal{Z} \times[0,1]$ where $K$ is an artificial bound, which will disappear in the limit as $h \rightarrow 0$ and $N h \rightarrow \infty$. For given $\hat{S}^{h}(0)$, define the Markov chain $\left\{\hat{S}^{h}(k): k=0,1, \cdots\right\}$ recursively by

$$
\hat{S}^{h}(k+1)=\hat{S}^{h}(k)+\hat{W}^{h}(k) \quad k=0,1, \cdots,
$$

where, for each $k$, given state $\hat{S}^{h}(k)=\hat{x}=\left(x_{0}, \cdots, x_{n+1}\right) \in \hat{\mathcal{H}}_{N}^{h}$ and control $\alpha:=(\hat{c}, \hat{l}, \hat{m}, \hat{u}) \in \hat{U}_{N}^{h}$, the (conditional) distribution of $\hat{W}^{h}(k)$ is denoted by $\hat{P}(\cdot \mid \hat{x}, \alpha)$, and is defined as follows. 
For $\hat{x} \in \hat{\mathcal{S}}_{N}^{h}$

$$
\begin{aligned}
& \hat{P}^{h}\left(e_{i} h \mid \hat{x}, \alpha\right)=\frac{\hat{u} a_{i i} x_{i}^{2} / 2-\hat{u} \sum_{j \neq i}\left|a_{i j} x_{i} x_{j}\right| / 2+h\left(b_{i} x_{i}^{+} \hat{u}+(1-\hat{u}) \hat{l}_{i}\right)}{Q^{h}(\hat{x})} \\
& \hat{P}^{h}\left(-e_{i} h \mid \hat{x}, \alpha\right)=\frac{\hat{u} a_{i i} x_{i}^{2} / 2-\hat{u} \sum_{j \neq i}\left|a_{i j} x_{i} x_{j}\right| / 2+h\left(b_{i} x_{i}^{-} \hat{u}+(1-\hat{u}) \hat{m}_{i}\right)}{Q^{h}(\hat{x})} \\
& \hat{P}^{h}\left(e_{0} h \mid \hat{x}, \alpha\right)=\frac{h\left(r x_{0}^{+} \hat{u}+(1-\hat{u}) \sum_{i=1}^{n}\left(1-\mu_{i}\right) \hat{m}_{i}\right)}{Q^{h}(x)} \\
& \hat{P}^{h}\left(-e_{0} h \mid \hat{x}, \alpha\right)=\frac{h\left(r x_{0}^{-} \hat{u}+(1-\hat{u}) \sum_{i=1}^{n}\left(1+\lambda_{i}\right) \hat{l}_{i}+\hat{c} \hat{u}\right)}{Q^{h}(\hat{x})}, \\
& \hat{P}^{h}\left(e_{n+1} h \mid \hat{x}, \alpha\right)=\frac{h \hat{u}}{Q^{h}(x)}, \quad i=1, \cdots, n, \\
& \hat{P}^{h}\left(e_{i} h \pm e_{j} h \mid \hat{x}, \alpha\right)=\frac{\hat{u}\left(a_{i j} x_{i} x_{j}\right)^{ \pm} / 2}{Q^{h}(\hat{x})} \quad i, j=1, \cdots, n \quad i \neq j \\
& \hat{P}^{h}\left(-e_{i} h \mp e_{j} h \mid \hat{x}, \alpha\right)=\frac{\hat{u}\left(a_{i j} x_{i} x_{j}\right)^{ \pm} / 2}{Q^{h}(\hat{x})} \quad i, j=1, \cdots, n \quad i \neq j, \\
& \hat{P}(0 \mid \hat{x}, \alpha)=1-\sum_{w \neq 0} \hat{P}(w \mid \hat{x}, \alpha) .
\end{aligned}
$$

The normalizing constant $Q^{h}(\hat{x})$ is taken so that for all $\alpha \in \hat{U}_{N}^{h}$

$$
\begin{array}{r}
Q^{h}(\hat{x}) \geq\left[\sum_{i=1}^{n} a_{i i} x_{i}^{2} \hat{u}\right. \\
-\sum_{i} \sum_{j}\left|a_{i j} x_{i} x_{j}\right| \hat{u} / 2+h\left\{\sum_{i=1}^{n}\left[b_{i}\left|x_{i}\right| \hat{u}+\left(\hat{l}_{i}+\hat{m}_{i}\right)(1-\hat{u})\right]\right. \\
\left.\left.+r\left|x_{0}\right| \hat{u}+\sum_{i=1}^{n}\left[\left(1+\lambda_{i}\right) \hat{l}_{i}+\left(1-\mu_{i}\right) \hat{m}_{i}\right](1-\hat{u})+\hat{c} \hat{u}+\hat{u}\right\}\right] .
\end{array}
$$

For $\hat{x} \in \partial \hat{\mathcal{S}}_{N}^{h}$

$$
\hat{P}(-\hat{x} \mid \hat{x}, \alpha)=1 \text {. }
$$

Hence $\partial \hat{\mathcal{S}}_{N}^{h}$ is an absorbing set for the chain, in fact $\{0\}$ is an absorbing state. This ensures that we obtain the correct boundary value for our approximation. For $\hat{x} \in \partial \hat{\mathcal{S}}_{N}^{h+}$

$$
\hat{P}(\hat{z}-\hat{x} \mid \hat{x}, \alpha)=1,
$$

where $\hat{z}$ is the point in $\hat{\mathcal{S}}_{N}^{h}$ nearest to $\hat{x}$. This makes $\partial \hat{\mathcal{S}}_{N}^{h+}$ reflecting.

We need to ensure that the above expressions are transition probabilities, i.e. we want

$$
\hat{u}\left[a_{i i} x_{i}^{2}-\sum_{j: j \neq i}\left|a_{i j} x_{i} x_{j}\right|\right] \geq 0, i=1, \cdots, n, x \in \mathcal{S} .
$$


To this end, define $\rho_{i}=a_{i i} / \sum_{j: j \neq i}\left|a_{i j}\right|, i=1, \cdots, n$, and let

$$
\mathcal{K}:=\left\{x \in \mathbb{R}^{n+2}: \rho_{i}^{-1} \leq\left|\frac{x_{i}}{x_{j}}\right| \leq \rho_{j}, i, j=1, \cdots, n\right\} .
$$

Here the $\rho$ 's may assume the value $\infty$. Now restrict $\hat{u}(\hat{x})=0$ for $\hat{x} \notin \mathcal{K}$. We are in fact forcing the system to trade into $\mathcal{K}$. From a heuristic analysis, cf [1], we expect that the no trade region is a cone in the first orthant. Our method requires that this cone be contained in $\mathcal{K}$. In practice, one proceeds under this assumption, and then checks at the end whether the boundary of $\mathcal{K}$ intersects the no trade region. If not, then all is well, but if it does, then another method must be found. We formalize this assumption as

- [A.3] $\mathcal{K}$ contains the no trade region.

Of course, if $a$ is diagonal then [A.3] holds.

Definition 3.1 A control $\hat{\mathcal{P}}^{h}=\left(\hat{\mathcal{P}}^{h}(k)\right)_{k=0}^{\infty}:=((\hat{c}(k), \hat{l}(k), \hat{m}(k), \hat{u}(k)))_{k=0}^{\infty}$ is said to be admissible for the Markov chain if

$$
\hat{S}(k) \in \hat{\mathcal{H}}_{N}^{h} \quad \text { a.s. for } k=0,1, \cdots
$$

The set of admissible controls is denoted by $\hat{\mathcal{U}}_{N}^{h}$.

For $\hat{x} \in \hat{\mathcal{H}}_{N}^{h}$ and $\hat{\mathcal{P}}^{h} \in \hat{\mathcal{U}}_{N}^{h}$, define

$$
\hat{J}^{h}\left(\hat{x}, \hat{\mathcal{P}}^{h}\right)=\hat{E}_{\hat{x}, \hat{\mathcal{P}}^{h}}\left[\sum_{k=0}^{\infty} e^{-\delta \hat{S}_{n+1}^{h}(k)} \frac{\hat{c}(k)^{\gamma}}{\gamma} \hat{u}(k) \Delta t^{h}\left(\hat{S}^{h}(k)\right)\right],
$$

where

$$
\Delta t^{h}(\hat{x})= \begin{cases}h^{2} / Q^{h}(\hat{x}) & \text { if } \hat{x} \in \hat{\mathcal{S}}_{N}^{h}, \\ 0 & \text { if } \hat{x} \in \partial \hat{\mathcal{S}}_{N}^{h+} \cup \partial \hat{\mathcal{S}}_{N}^{h}\end{cases}
$$

and $\hat{E}_{\hat{x}, \hat{\mathcal{P}}^{h}}$ is the expectation operator given $\hat{S}^{h}(0)=\hat{x}$ and control $\hat{\mathcal{P}}^{h}$. The value function is then defined to be

$$
\hat{V}^{h}(\hat{x})=\sup _{\hat{\mathcal{P}}^{h} \in \hat{\mathcal{U}}_{N}^{h}} \hat{J}^{h}\left(\hat{x}, \hat{\mathcal{P}}^{h}\right) .
$$

The problem is to find a control $\tilde{\mathcal{P}}^{h} \in \hat{\mathcal{U}}_{N}^{h}$ such that

$$
\hat{V}^{h}(\hat{x})=\hat{J}^{h}\left(\hat{x}, \tilde{\mathcal{P}}^{h}\right) \quad \forall \hat{x} \in \hat{\mathcal{S}}_{N}^{h} \cup \partial \hat{\mathcal{S}}_{N}^{h+} .
$$

Remark 3.1 If $\hat{x} \in \partial \hat{\mathcal{S}}_{N}^{h}$, then it remains there, so $\Delta t^{h}(\hat{S}(k))=0, k=0, \cdots$. This implies that $\hat{J}^{h}\left(\hat{x}, \hat{\mathcal{P}}^{h}\right)=0$ for any admissible control sequence $\hat{\mathcal{P}}^{h}$, which in turn implies that $\hat{V}^{h}(\hat{x})=0$. 
The following convergence result can now be established.

Theorem 3.1 Assume [A.1]-[A.3]. Then for $\hat{x} \in \overline{\hat{\mathcal{S}}}$

$$
\lim _{\substack{h \downarrow 0 \\ \hat{y}^{h} \rightarrow \hat{x} \\ y^{h} \in \mathcal{S}_{N}^{h}}} \hat{V}^{h}\left(\hat{y}^{h}\right)=\hat{V}(\hat{x})
$$

where $\hat{V}$ is the unique viscosity solution of (2.15).

\section{References}

[1] M. AKIAN, J. L. MENALDI, AND A. SULEM, On an investmentconsumption model with transaction costs, SIAM J. Control. Optim, 1 (1996) pp. 1-35.

[2] G. M. CONSTANTINIDES, Capital market equilibrium with transaction costs, J. of Political Economy, 94 (1986), pp. 842-864.

[3] M. H. A. DAVIS AND A. R. NORMAN, Portfolio selection with transaction costs, Math. Oper. Res, 15 (1990), pp. 676-713.

[4] B. G. FITZPATRICK AND W. H. FLEMING, Numerical methods for an optimal investment-consumption model, Math. Oper. Res, 16 (1991) pp. 823-841.

[5] U. G. HAUSSMANN AND W. SUO, Existence of singular optimal control laws for stochastic differential equations, Stochastics and Stochastics Reports, 48 (1994) pp. 249-272.

[6] I. KARATZAS, J. P. LEHOCZKY, S. P. SETHI AND S. E. SHREVE, Explicit solution of a general consumption-investment problem, Math. Oper. Res, 11 (1986), pp. 261-294.

[7] H. J. KUSHNER AND P. G. DUPUIS, Numerical methods for stochastic control problems in continuous time, Springer Verlang, New York, (1992).

[8] H. J. KUSHNER AND L. F. MARTIN, Routing and singular control for queuing networks in heavy traffic, SIAM J. Control, Optim, 28 (1990), pp. 1209-1233.

[9] M. J. MAGILL AND G. M. CONSTANTINIDES, Portfolio selection with transaction costs, J. Economic Theory, 13 (1976), pp. 245-263. 